\title{
The application of the principles of the theory of shakedown to the calculation of pavement layers of granular materials in shear
}

\author{
Anatoly Aleksandrov ${ }^{1}$, Gennadiy Dolgih ${ }^{1}$, Vitaly Ignatov ${ }^{1}$, and Aleksander Kalinin ${ }^{1 *}$ \\ ${ }^{1}$ Siberian State Automobile and Highway University (SibADI), pr. Mira 5, Omsk, 644080, Russia
}

\begin{abstract}
In the report ways of application of the theory of shakedown of material to calculation of coverings and the bases of pavements from the granulated materials are considered. The basic limits of shakedown are given, which include the limits of resilient, elastic and plastic shakedown. The results of three-axis tests are given, on the basis of which the character of accumulation of residual deformation is divided into damped, steady and progressive. These data allow to predict the nature of deformation and provide damped deformation of the granular material, limiting the stress at the most dangerous point.
\end{abstract}

\section{Introduction}

Abroad in order to calculate road structures began to apply the theory of shakedown of materials. And now this theory is becoming increasingly popular. According to the authors, this theory can be used in the calculation of granular materials used in the structural layers of pavement.

The theory of shakedown was proposed for metals and then applied to the calculation of structures. In the traditional understanding of the phenomenon of shakedown is that under the influence of cyclic loading plastic deformation disappears after the implementation of a certain number of repeated loads, resulting in further deformation of the material structure occurs in the resilient stage. O.F. Cherniavsky notes that the stable operation of the design occurs whith the same influence on the deformation of processes of tiredness and hardening $[1, \mathrm{p} .4]$. At the previous or subsequent stage of deformation prevails any one of these processes: hardening - at the stage of initial deformation, softening - at the stage of predestruction [1, p. 4].

Thus, the classical theory of shakedown focuses on stable elastic-plastic deformation, separating it from the previous and subsequent stages of hardening and pre-destruction (plastic or fragile). The theory is based on the static theorem of Melan [2, p. 338] and Koiter [2, p. 341], which allow to determine the lower and upper limits of shakedown. But these theorems do not allow us to determine the number of cyclic loads, at the implementation of which shakedown occurs, and the value of plastic deformation accumulated under initial deformation conditions.

\footnotetext{
* Corresponding author: alexsandr55ne@mail.ru
} 


\section{Materials and Methods}

In modern interpretation, the theory of shakedown includes several limits for stresses, the combinations of which limit the various stages of deformation, which precede or, conversely, are subsequent to the stable stage of deformation, considered in classical theory.

In accordance with the basic provisions of this theory, the resistance of the material to deformation has 3 [3] or 4 level's [4]: resilient (pure resilient or fully resilient) shakedown, elastic shakedown, plastic shakedown and destruction $[3,4]$. The limits of shakedown and the corresponding stages of deformation under cyclic influence of alternating load are shown in figure 1 .

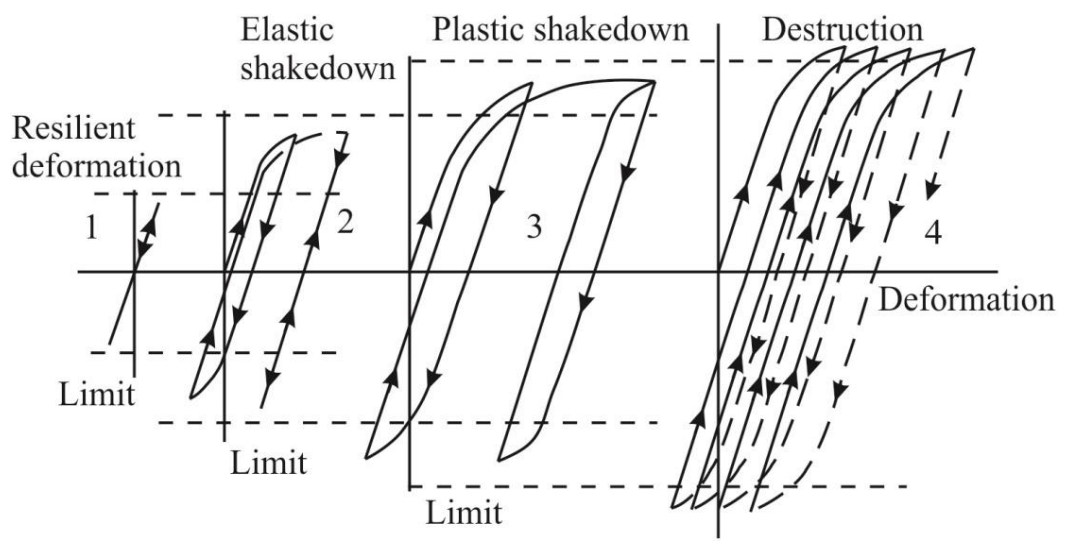

Fig. 1. Limits of shakedown theory [346]: $1-4$ - accordingly, the resilient, viscoelastic, elastoplastic damped and progressive deformation

Figure 1 shows the 4 different stages of deformation. The first stage of deformation is the stage of resilient deformations or, as they say, purely resilient deformations. Such deformation occurs when the stress state is so low under the influence of repeated loads that the structure of the material does not change. This stage of the material operation is typical for stresses not exceeding the resilient shakedown limit $[3,4]$. In this case, the material deformation graphs at application and removal of the load completely coincide, without forming a hysteresis loop. The maximum level of stress, at which such deformation occurs is called the limit of resilient shakedown [3]. This stage of loading the material is considered not by all authors [5].

In the case of a slight excess by the level of the stress state of this limit, the deformation is viscoelastic, that is reversible, but are restored only after a certain time after removal of the load $[3,4]$. In this case, a hysteresis loop is formed. With the implementation of repeated loads, the material adapts to this stress state and its deformations become purely resilient and hysteresis loops are no longer formed [3,6]. E. Rascol call this process - adaptation [5]. At this stage, there may be some plastic deformations, which, apparently, develop due to the compaction of mineral particles of the soil. However, such deformations stabilize and disappear after applied the first cycles of re-load.

The limit of elastic shakedown is the maximum level of the stress state at which the deformation of this nature is observed $[3,6]$. If the stress level exceeds this limit by an arbitrarily small amount, then part of the deformation will become plastic, including compaction and shear. However, in process of implementation of repeated loads, the material will adapt to such stresses, plastic deformation will become equal to zero, and the deformation will have an viscoelastic nature [3]. In the work of E. Rascol, this stage of 
deformation is called shakedown [5]. The maximum level of stress state for such deformation is called the limit of plastic shakedown $[3,6]$. A slight excess of this limit leads to a progressive plastic deformation, which, unlike the previous stages, cannot acquire the character of a damped plastic deformation, which ultimately ends in either the destruction of the material or failure as a result of the acquisition of an excessive deformation [7,8]. The limit value of the stress state level for this type of deformation is the limit of destruction [3, $7,8]$.

R.W. Sharp [9, 10] first applied the theory of shakedown in the design of road constructions. The basis for this was the data of M.F. Kent [11], according to which there was a stabilization of deformation in some road structures. Subsequently, the upper limit of the stress state level was established, at which at the first stage the material is compacted, experiencing sufficiently large deformations, but as the number of loads increases, the deformation increment decreases, tending to zero $[12,13]$. The same conclusions are made by J.H. Maree [14]. Research in the field of application of the theory of shakedown to soils and road structures includes numerical modeling and experimental determination of the limits of shakedown. The experiments are carried out as a full-scale test of models of road structures and also as three-axis compression of materials in the laboratory. The essence of these experiments is to analyze the dependencies of the deformations of the level of stress state and number of repeated loads.

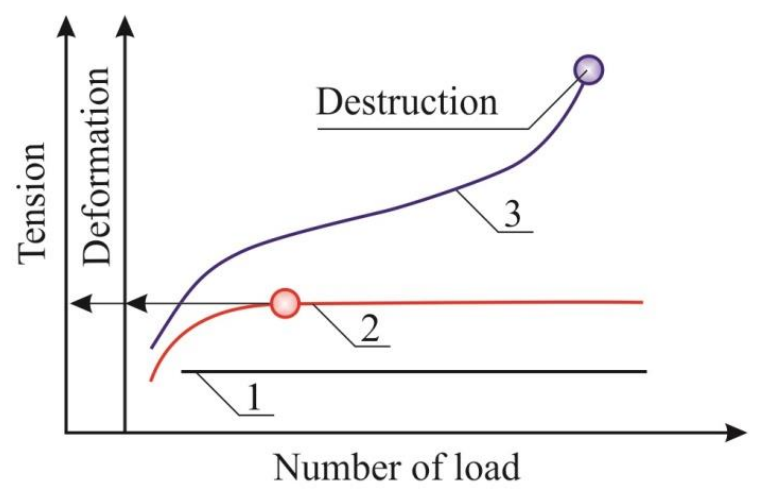

Fig. 2. Scheme for determining the limit of plastic deformation [15, p.3]: 1 - elastic deformation ; 2 elastic-plastic deformation with damped residual deformations, the stabilization of which determines the limit of plastic shakedown ; 3 - progressive elastic-plastic deformation

It follows from the analysis of the experimental results that it is quite difficult to establish the limits of shakedown in the form of absolute values, since the statement of the fact of absence of progressive character of deformation (figure 2, curve 3 ) requires the application of a large number of loads. At the same time, experts managed to establish conditional limits, which are physically similar to the limits of shakedown [3, 4]. In accordance with these works, the nature of the accumulation of plastic deformation is divided into three ranks: $A, B$ and $C[3,4]$. Rank $A$ - this is an irreversible deformation of materials, during which, as the number of loads increases, the residual deformation fades and eventually the material adapts to the stress state, having an increment of plastic deformation, tending to zero $[3,4]$. This deformation occurs when the difference between the residual deformations accumulated for 5000 and 3000 cycles is less than $0,04510^{-3}$, that is $\varepsilon_{5000}-\varepsilon_{3000}<0,04510^{-3}$ [3]. Deformation of rank $C$, on the contrary, they have a progressive character and occur when the difference of plastic deformations $\varepsilon_{5000}-\varepsilon_{3000}>0,410^{-3}$ [3]. For rank $B$ almost linear accumulation of plastic deformations to a sufficiently large number of applied loads is characteristic $(N=500000 \ldots 850000)$, and then $\varepsilon_{N}$ increases nonlinearly [3]. This type of deformation is 
determined by the condition under which the deformation difference must be $0,04510^{-}$ ${ }^{3}<\varepsilon_{5000}-\varepsilon_{3000}<0,410^{-3}[3]$.

Thus, thanks to the work of German specialists, the deformations are revealed, which allow to classify the character of plastic deformation into a damped, steady and progressive one. Subsequently S. Werkmeister, A. Dawson and F. Wellner it was proposed to determine the limit of plastic shakedown by deformations corresponding to the rank $A$ [6]. The possibility of this assumption is confirmed by experimental data, indicating that the limit number of loads, at the implementation of which there is a certain state of the material (stabilization of deformation or, conversely, an increase in the intensity of plastic deformation), depends on the magnitude of the main stresses and physical properties of the material under study. So, R.D. Barksdale [16], S.F. Brown [17], F. Lekarp, I.R. Richardson, A. Dawson [18], J.R. Morgan [19] agree that at a sufficiently low deviator residual deformation tends to a certain maximum value, and its increment-to zero. On the basis of these data, it was concluded that with a decrease in the deviator and an increase in the holding stress, the intensity of plastic deformation decreases. T. Wichtmann [20], R.D. Barksdale [21] it was reported that there is a certain threshold value of the deviator, in exceeding of which the increment of the residual deformation increases, that is, the nature of the deformation becomes progressive. In work R. Numrich and S. Werkmeister it is established that at three-axis tests the values of the maximum main stress correspond to the holding stresses at which the material under conditions of repeatedly applied loads experiences deformations rank $A, B$ or $C$. For deformations of all ranks, this relation of principal stresses is described by an exponential equation

$$
\sigma_{3}=A \cdot \exp \left(-B \cdot \frac{\sigma_{1}}{\sigma_{3}}\right),
$$

where $\sigma_{3}$ - limit for deformation of $\operatorname{rank} A, B$ or $C$ the value of the holding stress at a given $\sigma_{1}, \mathrm{kPa} ; A$ and $B$ - model parameter.

Note that for practical application the formula (1) is inconvenient, it is more rational to calculate the limit value for the maximum main stress. This can be done by solving expression (1) with regard $\sigma_{1}$.

$$
\sigma_{1 \lim }=-\frac{\sigma_{3}}{B} \cdot \ln \frac{\sigma_{3}}{A}
$$

Analysis of the formula (2) shows that the value of the coefficient $A$ should be less than $\sigma_{3}$. The values of the model parameters for the sand-gravel mixture are given in table 1 .

Table 1. Values of model parameters (1) and (2) for sand-gravel mixture with humidity $4 \%$ [6]

\begin{tabular}{|c|c|c|}
\hline \multirow{2}{*}{ Rank of deformation } & \multicolumn{2}{|c|}{ Value of the parameters } \\
\cline { 2 - 3 } & 765,5 & $B$ \\
\hline$A$ & 791,96 & 0,4564 \\
\hline$B$ & 771,63 & 0,3466 \\
\hline$C$ & & 0,2118 \\
\hline
\end{tabular}

\section{Results}

Note that the expressions (1) and (2) allow us to formulate the condition of the limiting state at shakedown in the form of a part of the surface located in the quadrant of compressive stresses or criterion. The equations describing such a surface and the criterion have the form: 


$$
\begin{gathered}
\sigma_{1}+\frac{\sigma_{3}}{B} \cdot \ln \frac{\sigma_{3}}{A}=0 . \\
-\frac{\sigma_{3}}{\sigma_{1}} \cdot \ln \frac{\sigma_{3}}{A}=B .
\end{gathered}
$$

According to the authors, the criterion (4) is of practical interest, as it allows to limit the level of stress state at the most dangerous point, taking into account the necessary value of the accumulated plastic deformation. In addition to the empirical approach R. Numrich and S. Werkmeister there are works that aim to link the number of loads, after which comes the elastic or plastic shakedown with the parameters of the limit straight Coulomb-Mohr.

Thus, by limiting the magnitude of the main stresses by limits of shakedown, it is possible to perform calculations of road structures according to the criteria:

- tangential stress whose magnitude at the most dangerous point is limited to the value of the corresponding limit of shakedown;

- pressure transmitted by the pavement to the roadbed, at which in the most dangerous point there is a limit state characterizing the occurrence of elastic or plastic shakedown.

To calculate thetangential stress, we apply the plasticity condition proposed by us in paper [22]. The limiting state of this condition of plasticity is described by the equation:

$$
\frac{1}{2} \cdot\left(\sigma_{1} \cdot\left(\frac{1-\sin \varphi}{1+\sin \varphi}\right)^{d}-\left(\frac{1+\sin \varphi}{1-\sin \varphi}\right)^{d} \cdot \sigma_{3}\right)=c .
$$

where $\sigma_{1}$ and $\sigma_{3}$ - maximum and minimum main stresses, $\mathrm{kPa} ; \varphi$ - angle of internal friction, degrees; $\mathrm{c}$ - cohesion, $\mathrm{kPa}$; $\mathrm{d}$ - the third parameter of the material, introduced by us in order to the value of the tangential stress determined by the left side of the equation (5) can vary from the values corresponding to the original Coulomb - Mohr criterion $(d=0.5)$ to the value corresponding to the third theory of strength $(\mathrm{d}=0)$.

Considering the normative method of calculating the pavement on shear in the soil and pavement layers from loosely coupled materials [23], and given that in this method the calculation of tangential stress can be made by left side of the dependence (5) we obtain:

$$
\frac{1}{2} \cdot\left(\sigma_{1} \cdot\left(\frac{1-\sin \varphi_{N}}{1+\sin \varphi_{N}}\right)^{d}-\left(\frac{1+\sin \varphi_{N}}{1-\sin \varphi_{N}}\right)^{d} \cdot \sigma_{3}\right)=T_{\lim },
$$

where $c_{N}$ and $\varphi_{N}$-angle of internal friction after application of the $N$-th number of design loads, degree; $T_{\mathrm{lim}}$ - maximum shear resistance at the most dangerous point, $\mathrm{kPa}$;

The limit shear resistance will be calculated by the formula ODN 218.046-01 [23], but adjusted for the fact that the most dangerous point is located at some distance from the surface of the roadbed. In this case, the formula takes the form:

$$
T_{\text {lim }}=c_{N} \cdot k_{\mathrm{d}}+\left(\sum_{i=1}^{n} \gamma_{i} \cdot h_{i}+\gamma_{i+1} \cdot z_{\text {sur }}\right) \cdot \operatorname{tg} \varphi_{1},
$$

where $\varphi_{1}-$ angle of internal friction at a single applied load, degree; $k_{\mathrm{d}}$ - coefficient taking into account the peculiarities of the structure on the border of the sand layer with the lower layer of the bearing base (accepted as directed [23]); i and $n-$ number and total number of layers of pavement; $\gamma_{i}$ - weighted average specific gravity of the $\mathrm{i}$ - th structural layer located above the tested layer, $\mathrm{kN} / \mathrm{m}^{3} ; h_{i}$ - thickness of the i-th layer of pavement; $\gamma_{i+1}-$ weighted average specific gravity of the soil of the subgrade or the material of the tested layer in the area above the test point, $\mathrm{kN} / \mathrm{m}^{3} ; z_{\text {sur }}$ - distance from the surface of the roadbed to the most dangerous point, $\mathrm{m}$; 
Since the formula (2) allows to calculate the limit value of the maximum main stress at a given value of the minimum main stress, it can be used in the left part of the plasticity condition (6). By substituting the expression (2) in the left part of equation (6) we obtain:

$$
\frac{1}{2} \cdot\left(-\frac{\sigma_{3}}{B} \cdot \ln \frac{\sigma_{3}}{A} \cdot\left(\frac{1-\sin \varphi_{N}}{1+\sin \varphi_{N}}\right)^{d}-\left(\frac{1+\sin \varphi_{N}}{1-\sin \varphi_{N}}\right)^{d} \cdot \sigma_{3}\right)=T_{\lim }
$$

It follows from the analysis of criterion (8) that for the deformation of a material or soil with the required deformation rank, the tangential stress determined by the left part of this equation is a certain limit value depending on the minimum principal stress. Moreover, the higher the value of the minimum main stress $\sigma_{3}$, the greater the value of the limit value of the maximum main stress $\sigma_{1 \mathrm{lim}}$, calculated by the formula (2). This leads to the fact that at a given value of the minimum main stress $\sigma_{3}$, there is an increase in both $\sigma_{1 l i m}$ in the formula (2) and an increase in the tangential stress in the left part of the dependence (8). As the angle internal friction increases, the tangential stress determined by the left side of equation (8) decreases. Other things being equal, the lowest values of tangential stress occur when $d=0.5$. In this case, their value corresponds to the tangential stresses of the Coulomb-Mohr plasticity condition with the difference that the maximum principal stress is calculated by the formula (2).

Since the tangential stresses calculated in the left side of equation (8) must be equal to the limit value, which is calculated by the dependence (7), both formulas can be equated. As a result, we obtain:

$$
\frac{1}{2} \cdot\left(-\frac{\sigma_{3}}{B} \cdot \ln \frac{\sigma_{3}}{A}\left(\frac{1-\sin \varphi_{N}}{1+\sin \varphi_{N}}\right)^{d}-\left(\frac{1+\sin \varphi_{N}}{1-\sin \varphi_{N}}\right)^{d} \cdot \sigma_{3}\right)=c_{N} k_{\mathrm{d}}+\left(\sum_{i=1}^{n} \gamma_{i} h_{i}+\gamma_{i+1} \cdot z_{\text {sur }}\right) \operatorname{tg} \varphi_{1} .
$$

By solving equation (9) with respect to the pressure transmitted by the layers of the pavement, located above the layer under test, on this layer, we obtain:

$$
\sum_{i=1}^{n} \gamma_{i} h_{i}=\frac{1}{\operatorname{tg} \varphi_{1}} \cdot\left[\frac{1}{2} \cdot\left(-\frac{\sigma_{3}}{B} \cdot \ln \frac{\sigma_{3}}{A}\left(\frac{1-\sin \varphi_{N}}{1+\sin \varphi_{N}}\right)^{d}-\left(\frac{1+\sin \varphi_{N}}{1-\sin \varphi_{N}}\right)^{d} \cdot \sigma_{3}\right)-c_{N} k_{\mathrm{d}}\right]-\gamma_{i+1} \cdot z_{\text {sur }}
$$

\section{Conclusion}

From the analysis of dependence (10) it follows that by varying the thickness of one or several layers of pavement it is possible to ensure the fulfillment of criterion (10), and hence the conditions (8) and (9). Note that to calculate the magnitude of the minimum main stress is impossible to apply the formula of soil mechanics [24-26]. The fact is that these dependences do not take into account the deformation characteristics of the material and the final thickness of the layers of the pavement. To calculate the minimum main stress, it is advisable to apply the proposed by us method published in [27] for the half-space and [28] for the layer of finite thickness.

Of course, from the standpoint of improving the evenness of the pavement, the application of the theory of shakedown opens up great prospects. However, the dependence (1) on which all our calculations are based is an empirical formula that has its own boundary conditions. Beyond these conditions in criteria (8) - (10) can't go out. For various granular materials, formulas similar to dependence (1) are known, they also have boundary conditions. Therefore, for the practical application of dependence (10) it is necessary to extend the 
boundary conditions of empirical formulas similar to the expression (1). This requires experimental research, requiring the use of dynamic triaxial compression devices, allowing to apply multiple deviatoric loads of different trajectories.

\section{References}

1. O.F. Cherniavsky Bulletin YuUrGU T13 2(4) (2013)

2. L.M. Kachanov Fundamentals of the theory of plasticity (Science, Moscow, 1969)

3. S. Werkmeister Permanent deformation behavior of unbound granular materials in pavement constructions (Ph.D. thesis, University of Technology, Dresden, 2003)

4. R. Numrich Modellierung des nichtlinear-elastischen verformungsverhaltens von tragschichten ohne bindemittel (Dissertation Von der Fakultat Bauingenieurwesen der Technischen Universitat Dresden zur Erlangung der Wurde eines Doktor-Ingenieurs (Dr.-Ing.) genehmigte 2003)

5. E. Rascol Cyclic Properties of Sand: Dynamic Behaviour for Seismic Applications (PhD thesis, at Laboratoire de mécanique des sols, École Polytechnique Fédérale de Lausanne, Lausanne, 2009)

6. S. Werkmeister, A. Dawson, F. Wellner Road Materials and Pavement Design, 6 (1) 31 (2005)

7. K.L. Johnson Inelastic contact: Plastic flow and shakedown (In $1^{\text {st }}$ International Conference of Contact Mechanics and Wear of Rail/Wheel Systems, Vancouver, Canada, University of Waterloo Press 79 (1982)

8. K.L. Johnson Plastic Flow, residual stresses and shakedown in rolling contact (Proceedings of the 2nd International Conference on Contact Mechanics and Wear of Rail Wheel Systems, University of Rhode Island, Waterloo Ontario, 1986)

9. R.W. Sharp Shakedown-Analyses and the design of pavement under moving surface load (PhD Thesis, University of Sydney, Australia,1983)

10. R.W. Sharp, J.R. Booker Journal of Transportation Engineering. 110, 1 (1984)

11. M.F. Kent AASHO road test vehicle operating costs related to gross weight (Highway Research Board, Special Report 73, 1962)

12. I.F. Collins, P.F. Cliffe International Journal for Numerical and Analytical Methods in Geomechanics, 11(4) 409 (1987)

13. I.F. Collins, A.P. Wang Saunders L.R. Australian Road Research Board, Road and Transport Research 2(4), 28 (1993)

14. J.H. Maree, C.R. Freeme, N.J. Van Zyl, P.F. Savage The permanent deformation of pavements with untreated crushed stone bases as measured in heavy vehicle simulator tests Proc. 11th ARRB Conf. part2 16 (1982)

15. S. Juspi Experimental validation of the shakedown concept for pavement analysis and design (PhD thesis University of Nottingham 2007)

16. R.D. Barksdale Laboratory Evaluation of Rutting in Base course Materials (Proceedings of the 3-rd International Conference on Asphalt Pavements, London 161 1972)

17. S.F. Brown Journal of Geotechnical Engineering Division, ASCE 100 (7) 825 (1974)

18. F. Lekarp, I.R. Richardson, A. Dawson Influences on Permanent Deformation Behavior of Unbound Granular Materials (Transportation Research Record, 1547, Transportation Research Board, Washington, 68 1996) 
19. J.R. Morgan The Response of Granular Materials to Repeated Loading, Proc. 3rd Conf. ARRB 1178 (1966)

20. T. Wichtmann, A. Niemunis, TH. Triantafyllidis Strain Accumulation in Sand due to Drained Uniaxial Cyclic Loading. Cyclic Behaviour of Soils and Liquefaction Phenomena, Proc. of CBS04 Bochum, 233 (2004)

21. R.D. Barksdale Performance of Crushed-Stone Base Courses. (Transportation Research Board, Washington 78 1984)

22. A.S. Aleksandrov, A.L. Kalinin Engineering and construction magazine 7(59) 4 (2015)

23. ODN 218.046-01. Design of non-rigid road clothes (GSDH of Ministry Of Transport, Moscow, 2001)

24. R.G. Ahlvin, H.H. Ulery Highway Research Record Bull. 3421 (1962)

25. C.R. Foster, R.G. Ahlvin Highway Research Board vol. 33. 236 (1954)

26. B.M. Das. Advanced soil mechanics, Third Edition (Taylor \& Francis, New York, 2008)

27. A.S. Aleksandrov, N.P. Aleksandrova, G.V. Dolgih Construction Materials, 10, 14 (2012)

28. A.S. Aleksandrov, G.V. Dolgih, A.L. Kalinin, Engineering and construction magazine 2(62) 51 (2016) 